Mots. Les langages du politique

Les élections législatives portugaises de 2002 et 2009 à partir des affiches politiques. Un « regard » multimodal

Rosalice B. W. S. Pinto

\title{
CpenEdition
}

Journals

Édition électronique

URL : https://journals.openedition.org/mots/21130

DOI : $10.4000 /$ mots. 21130

ISSN : 1960-6001

Éditeur

ENS Éditions

Édition imprimée

Date de publication : 22 avril 2013

Pagination : 47-60

ISBN : 978-2-84788-393-0

ISSN : 0243-6450

Référence électronique

Rosalice B. W. S. Pinto, « Les élections législatives portugaises de 2002 et 2009 à partir des affiches politiques. Un « regard » multimodal », Mots. Les langages du politique [En ligne], 101 | 2013, mis en ligne le 22 avril 2015, consulté le 23 avril 2022. URL : http://journals.openedition.org/mots/21130 ; DOI : https://doi.org/10.4000/mots.21130 


\section{Les élections législatives portugaises de 2002 et 2009 à partir des affiches politiques. Un « regard » multimodal}

Dans les pratiques sociales actuelles, lorsqu'on aborde les textes empiriques qui circulent, un premier constat général s’impose : ils appartiennent à des genres textuels divers, qui se donnent à voir sous de multiples formes. Par exemple, dans l'activité politique et plus précisément dans les campagnes législatives ou/et présidentielles, on a affaire à des objets complexes, constitués de textes écrits/oraux/verbaux et non verbaux. Par conséquent, l'étude de l'omniprésence de ces différentes composantes dans certaines activités sociales pose des exigences aussi bien théoriques que méthodologiques.

En s'inspirant d'une méthodologie descendante d'analyse, comme le préconise l'interactionnisme socio-discursif (Bronckart, 2008) et des travaux sur la sémiotique visuelle de Kress et Van Leeuwen (2006), ce travail se propose d'abord d'analyser quelques éléments multimodaux présents dans les affiches politiques. Ensuite, l'objectif est de montrer que l'organisation de ces éléments dans l'espace de la composition visuelle ainsi que les conduites affectives, relationnelles ou cognitives engendrées par l'image peuvent constituer des stratégies argumentatives importantes pour la visée persuasive de ce genre textuel.

Nous commencerons, sur le plan théorique, à pointer dans la littérature scientifique quelques travaux relatifs aux ressources multimodales mobilisées dans différents contextes et aux divers codes sémiotiques présents dans les textes. Ensuite, nous présenterons l'approche méthodologique adoptée : une méthodologie descendante d'analyse. Cela veut dire que les aspects multimodaux ou pluri-sémiotiques des textes sont forcément contraints par des aspects d'ordre générique. Ensuite, nous passerons à l'analyse des affiches. Les données empiriques sur lesquelles nous nous fonderons émanent de quelques exemples d'affiches politiques du Parti socialiste (dorénavant PS) qui ont circulé au Portugal pendant les élections législatives en 2002 et $2009^{1}$.

1. António Manuel de Oliveira Guterres, secrétaire général du Parti socialiste, est devenu Premier 


\section{Les approches multimodales}

Le champ des approches multimodales n'est pas un domaine bien défini. Inspiré des travaux pionniers de Roland Barthes développés en France pendant les années soixante et soixante-dix sur les images photographiques, les recherches contemporaines sur la question des interrelations entre différents systèmes d'expression dépassent une conception verbocentrique de l'interaction pour prendre en compte plusieurs modalités productrices de sens. De toute façon, il est important de souligner que ces approches abordent la communication comme un processus sémiotique global qui peut utiliser une combinaison de plusieurs systèmes de signes.

Les travaux pionniers sur la multimodalité décrivaient la multicanalité et les caractéristiques des unités dites para-verbales (les gestes, les mouvements et les postures) en prenant souvent la linguistique comme modèle pour leur découpage et leur analyse ${ }^{2}$. En revanche, actuellement, les approches contemporaines ont plutôt tendance à considérer que chaque mode sémiotique a ses spécificités descriptives. Par conséquent, deux grandes tendances 3 peuvent être observées. D'une part, quelques études, héritières de la sémiotique développée en France pendant les années soixante/soixante-dix, abordent la question des interrelations entre différents systèmes d'expression dans le cadre d'une sémiotique sociale plus large. D'autre part, d'autres approches tentent de décrire les spécificités et le fonctionnement de chaque système sémiotique individuel tel qu'il a été culturellement, socialement façonné.

Cependant, ce sont les travaux de Kress et Van Leeuwen (2006) ou de Kress (1998) relevant de la deuxième logique qui nous intéressent dans cette contribution. Ces auteurs analysent les éléments scripto-visuels 4 qui composent les images présentes dans plusieurs documents (affiches, publicités, manuels scolaires, livres d'enfants ou revues illustrées, etc.). Ils s'interrogent sur la nature, la signification de ces éléments (la taille, la couleur, la typographie) et sur leur organisation dans l'espace de la communication visuelle. S'inspirant des travaux de Michael Halliday (1985) sur la sémiotique sociales, ces

ministre en 1995; réélu en 1999, il est resté en fonction jusqu'en 2001. José Sócrates Carvalho Pinto de Sousa, l'autre Premier ministre socialiste, est resté au pouvoir de 2005 à 2011.

2. Dans cette perspective, nous considérons les travaux de Hall (1978) et de Brossard et al. (1984) cités dans De Saint-Georges (2008).

3. Cette position est soutenue par Lemke (1998) cité dans De Saint-Georges (2008, p. 120).

4. Comme nous privilégions dans cette contribution le travail de Kress et Van Leeuwen (2006) sur la sémiotique visuelle pour la description de la multimodalité, nous utilisons le terme scriptovisuel au lieu de scripto-iconique.

5. La sémiotique sociale envisage la dimension sociale des systèmes sémiotiques comme une donnée intrinsèque par rapport à leur nature et à leur fonction : décrire un langage implique donc simultanément la description des usages sociaux auxquels se prête ce langage. Pour des détails sur cette question, voir Van Leeuwen (2008, p. 47). 
auteurs présentent un inventaire des composantes majeures de l'image, c'està-dire celles qui ont été établies au fil de l'histoire des sémiotiques visuelles dans le monde occidental. Leurs études portent, d'une part, sur le système de choix dont disposent le producteur/le récepteur pour la production/réception des compositions visuelles et, d'autre part, sur les différences que ces choix peuvent produire en termes de signification les uns par rapport aux autres.

Il est important de mentionner que les travaux de Gunther Kress et de ses collègues proposent des catégories d'analyse qui, au niveau épistémologique, bouleversent les approches traditionnelles des études discursives. Ils remettent en cause l'arbitraire du signe ${ }^{6}$ et privilégient une réflexion sur les signes et leur perpétuelle transformation (en fonction des influences culturelles et sociales).

Dans leur proposition d'analyse, Kress et Van Leeuwen (2006) adoptent le concept théorique de Halliday pour l'étude des métafonctions, mais l'adaptent au mode sémiotique visuel. De cette façon, les métafonctions deviennent respectivement pour ces auteurs des signifiés représentationnels, interactifs et compositionnels. Et pour chaque sous-ensemble est développée une grille conceptuelle d'analyse.

Nous insistons sur le fait que, dans la perspective méthodologique interactionniste sociale privilégiée dans ce travail, les unités verbales et non verbales sont socialement dépendantes et interagissent constamment. Ainsi, lors de la production ou de l'interprétation, les agents impliqués peuvent (ré)actualiser les informations selon des contextes divers. Et c'est exactement dans cette optique, en intégrant des approches théoriques différentes mais complémentaires, de notre point de vue, que nous tenterons, dans cette contribution, d'analyser les aspects multimodaux présents dans les affiches politiques des campagnes législatives électorales 2002 et 2009.

Avant de commencer l'analyse des textes choisis, nous allons brièvement présenter quelques aspects importants du PS portugais ainsi que la situation économique et politique du Portugal en 2002 et 2009.

\section{Le Parti socialiste et le contexte politico-social portugais}

Quoique nous nous centrions surtout sur la description scripto-visuelle de quelques affiches politiques du PS portugais pendant la dernière décennie, ce qui peut attester aussi de son évolution idéologique au cours de cette période,

6. Selon Kress et Van Leeuwen (2006, p. 6), «the sign is not the pre-existing conjunction of a signifier and a signified, to be recognized and used en bloc, in the way that signs are usually defined in 'semiology', but a process of sign-making in which the stratum of the signifier and the stratum of the signified are relatively independent of each other... In our view signs are never arbitrary, and 'motivation' should be formulated in relation with the sign-maker and the context in which the sign is produced, and not in isolation from the act of producing analogies and classifications». 
il faut tisser d'abord quelques considérations sur l'origine de ce parti au Portugal avant de passer au contexte portugais de 2002 et 2009.

Le PS portugais est créé en 1973, en Allemagne de l'Ouest. Un des fondateurs du parti, Mário Soares, rentre au Portugal après la Révolution des Fillets en 1974 et occupe le poste de secrétaire général du parti pendant treize ans. Les premières élections législatives, en 1976, sont remportées par Mário Soares qui devient le premier chef de gouvernement socialiste du Portugal. Il reste au pouvoir de 1976 à 1978, puis de 1983 à 1985. D’autres secrétaires généraux du parti ont occupé le poste de Premier ministre : António Guterres (1995-2002) et José Sócrates (2005-2011).

Cette brève présentation de la présence du parti au Portugal met en évidence deux aspects importants. Tout d'abord, la jeunesse du PS portugais, de création plus récente que la majorité des autres partis socialistes en Europe (la fondation du PS français, par exemple, remonte à 1969), et aussi une certaine prédominance idéologique socialiste au Portugal. Depuis sa fondation, le PS est resté 17 ans au pouvoir.

Sans accorder trop de poids à ces observations préalables sur l'histoire du parti, nous porterons notre attention maintenant sur le contexte sociopolitique du Portugal pendant les années 2002 et 2009 . Comme le rappelle Patrick Charaudeau (2008, p.13), " une campagne électorale se fait à l'intérieur d'un espace public qui est socialement et politiquement organisé». Cela revient à dire que, pour pouvoir analyser les textes qui circulent à l'occasion d'un événement politique particulier, il faut que nous tenions compte de la situation sociale du pays à l'époque des élections et du contexte politique à partir duquel ils surgissent et se construisent. C'est à partir des corrélations établies entre ces divers éléments que nous pouvons comprendre les stratégies linguisticotextuelles utilisées par les partis politiques pour atteindre leur but : la victoire de leur candidat.

En décembre 2001, le Premier ministre portugais, António Guterres7, du PS, renonce à son poste après six ans de gouvernement. À l'époque, sa décision a été justifiée par la défaite du parti aux élections municipales réalisées quelques mois avant et la victoire du centre-droit à Lisbonne. Après avoir vécu plusieurs années de forte croissance, le Portugal a connu une contraction de son activité économique. Ces changements ont provoqué l'anticipation des élections législatives au Portugal. Les deux principaux partis, le PS et le PSD (Parti social-démocrate), ont choisi respectivement comme tête de liste

7. On doit souligner que l'évolution sociopolitico-économique du pays observée jusqu'à 2001 est visible sur les affiches politiques du PS qui ont circulé en 1992 et 1999. Le gouvernement de Guterres modernise l'image du parti. La rose est introduite en 1992 et apparaît à gauche du poing serré. Et, en 1995, un des plus grands publicitaires portugais - Edson Athayde - devient le responsable du marketing politique du parti. Les relations entre le chef du parti et un professionnel du marketing renforcent le caractère professionnel de la communication politique du PS. 
Eduardo Ferro Rodrigues et José Manuel Durão Barroso. Le premier avait été ministre de l'Équipement dans le gouvernement de Guterres et était peu connu du grand public - ce qui explique la présence fréquente, dans les affiches politiques, de la photo du candidat. Le second présidait déjà le PSD et, comme il était opposant au parti qui gouvernait à l'époque, son apparition dans les médias était habituelle. Les élections ont vu la victoire, en mars 2002, du PSD représenté par Durão Barroso.

Les élections législatives portugaises de 2009 se sont tenues en septembre. Le PS remporte le scrutin mais perd la majorité absolue (lors des élections législatives antérieures de 2005, le PS de José Sócrates avait remporté $45 \%$ des voix et 121 députés). Le gouvernement dirigé par le socialiste José Sócrates depuis 2005 débute son second mandat. En 2009, le Portugal souffre, depuis sa dernière récession en 2002, d'une croissance relativement médiocre avec une faible compétitivité, d'une sous-qualification de la main-d'œuvre spécialisée, d'une mauvaise gestion des finances publiques et d'une grande dépendance à l'égard de ses principaux partenaires commerciaux européens. Pour affronter ces questions, le programme électoral du PS table avant tout sur la réduction des dépenses d'investissements publics et insiste sur la nécessité de consolider les comptes publics.

Après ce bref survol du contexte politique des années étudiées, nous passerons à l'analyse de la dynamique multimodale des textes choisis. Comme nous le constaterons, ces changements contextuels laisseront des traces profondes dans l'organisation multimodale des affiches politiques analysées.

\section{L’affiche politique : un genre textuel?}

L'affiche politique se présente comme un genre textuel persuasif8 ${ }^{8}$ complexe qui vise à convaincre un électeur de voter pour un parti ou pour un candidat. Tous, à partir des modèles de texte intériorisés (dimension architextuelle) de ce qu'est une affiche politique, peuvent reconnaître ce genre et l'interpréter comme tel. Évidemment, les ressources multimodales utilisées dans les affiches sont contraintes par des aspects contextuels divers.

Du point de vue de la dimension spatiale, pour Patrick Charaudeau (1991, p.46), la communication par l'affiche suppose la restitution d'un espace tridimensionnel à l'intérieur d'une surface bidimensionnelle et cela implique une construction spatiale relativement complexe. N'oublions pas que l'affiche est construite de manière à être lue même lorsque le destinataire se trouve à distance et doit respecter des habitudes culturelles de construction et de perception. C'est dans cet espace complexe que la multimodalité matérialisée par

8. Pour la description de ces genres textuels, voir Pinto (2010). 
des modes de communication divers (aspects verbaux et non verbaux - graphisme, photographie, dessin, chromatisme) se construit.

En outre, les affiches circulent dans des endroits variés, en respectant la législation du pays. De cette façon, le lieu de circulation établi peut contraindre le choix des unités scripto-visuelles utilisées. Par exemple, l'affiche politique, ici au Portugal, est installée dans des espaces bien restreints : surtout les ronds-points, parce que la vitesse des voitures y est plus réduite. Les énoncés verbaux doivent par ailleurs être courts de manière à être lus facilement. Ils doivent se distinguer par leur dimension et leur aspect typographique. L'image doit, quant à elle, être présentée de manière à établir une interaction dont le "spectateur», placé à une certaine distance communicative, est le partenaire.

Les affiches s'inscrivent aussi dans une temporalité qui comporte plusieurs phases. Par exemple, la première vague d'affiches du Parti socialiste portugais en 2002 ne comporte que des prédicats moraux attribués au candidat de l'époque - coragem, determinação, seriedade (courage, détermination, sérieux) ; alors qu'à la dernière vague, elles contiennent un acte directif : «Vote em um Portugal solidário » («Votez pour un Portugal solidaire »).

Un autre aspect important à souligner est l'intertextualité qui caractérise l'élaboration et l'interprétation des affiches. Comme l'affirme Charaudeau (1991, p. 49), «la signification de l'affiche n'est pas dans l'affiche ». Le concept d'intertextualité rend compte de la dimension culturelle de tout segment de production qui n'a de sens que dans une historicité des manières de produire et de signifier. Dans la première affiche, par exemple, le lexème coragem ne peut être interprété qu'en connaissant de près la situation sociale portugaise à l'époque. Le Parti socialiste a été à l'initiative de l'ouverture des pharmacies populaires, ce qui représentait une décision importante pour la population.

\section{Méthodologie d'analyse}

La présente étude compare les affiches politiques du parti socialiste à deux époques bien différentes de son histoire : les années 2002 et 2009 . Pour ce faire, nous avons constitué un ensemble composé de six affiches de 2002 et cinq de 2009. Puis nous avons choisi un exemplaire prototypique 9 du genre (voir en annexe) ${ }^{10}$, que nous appellerons étude de cas. Ces textes présentent la ressemblance de famille ${ }^{11}$ du genre auquel ils appartiennent. Cette démarche

9. Terme emprunté aux travaux en sémantique et en psychologie. Georges Kleiber (1990) systématise beaucoup de ces travaux.

10. Voir: http://politikaeimagens.blogspot.pt/2009/09/legislativas-2009-ps_27.html.

11. Terme emprunté à George Lakoff. Selon cet auteur, cela correspond à «l'idée que les membres d'une catégorie peuvent être reliés les uns aux autres sans qu'ils aient une propriété en commun qui définisse la catégorie» (Lakoff, 1987, p.12, cité dans Kleiber, 1990, p.151). 
méthodologique souligne la complexité de notre objet d'étude (le texte comme unité communicative globale) et fournit des pistes intéressantes transférables à un corpus plus ample.

Observons maintenant la signification de la multimodalité dans les textes choisis et son rapport avec le moment socio-économique du pays.

\section{Étude de cas 1}

Ce texte est facilement identifiable comme appartenant au genre affiche politique (voir image 1 en annexe). D'emblée, nous pouvons distinguer des éléments verbaux et des éléments non verbaux. D'une part, le lexème coragem, délimité par un point final, apparaissant en haut de l'affiche, en caractères majuscules, dont la typographie (la graisse et la chasse) est remarquable, suivi du nom du candidat vers le milieu de l'image formé de caractères plus petits avec les initiales en majuscule; d'autre part, les éléments non verbaux. Nous observons la photographie du candidat Ferro Rodrigues au premier plan : costume sombre, lunettes carrées, cheveux grisâtres, sourire à peine esquissé. Nous observons aussi les deux symboles du parti : la rose et le poing serré. Nous repérons la mention du site internet du parti et enfin l'arrière-plan chromatique : ce sont les couleurs du drapeau portugais (vert et rouge foncés), présentées en dégradé.

Il importe de souligner que, pour ces élections de 2002, ce sont des individus de la base du parti et le secrétaire général qui se sont occupés de la communication politique. Le spécialiste en marketing politique de la campagne électorale de Guterres a été consulté mais il n'a pas eu à l'époque la même autonomie qu'auparavant (Lisi, 2008, p. 514).

Ce texte a une visée persuasive bien précise : obtenir le vote de la population pour le PS. Mais comment cette argumentation est-elle construite à l'intérieur de cet espace textuel?

Confronté à un tel ensemble, le regard de l'électeur peut parcourir divers trajets sur la surface de l'image et pourra, selon l'individu, les reconstruire de plusieurs façons. Nous essayerons maintenant de reconstituer l'un des trajets possibles et de vérifier les effets produits par quelques éléments verbaux et non verbaux de manière à prouver que l'acte directif centré sur le pathos - Votez pour le candidat, qui correspond à la thèse de l'affiche - est atteint à partir d'un choix rhétorique bien défini.

D'abord, le regard du spectateur parcourt le lexème coragem qui renvoie à la photographie du candidat. Nous pouvons associer l'un à l'autre et conclure : [Si je veux quelqu'un de courageux, il faut que je vote pour ce candidat]. Il se produit alors une sorte de dédoublement de cette image ou, comme le souligne Roland Barthes (1964, p.32), «le texte dirige le lecteur entre les signifiés de l'image, lui en fait éviter certains et en recevoir d'autres ». De cette manière, 
une polysémie iconique possible est évitée. Autrement dit, la signification du terme coragem dans cette affiche est éclairée par la liaison établie par le lecteur entre les signes verbaux et non verbaux du document. Mais ce qui nous touche surtout, c'est la manière plastique dont ce lexème est présenté. Les caractères majuscules, en gras, qui suivent une orientation verticale, renforcent le contenu sémantique du lexème coragem et attestent la vertu du candidat dont la photographie est présentée.

En parcourant l'image, nous remarquons le nom propre Ferro Rodrigues, formé de caractères sensiblement plus petits. Ce nom fonctionne comme une espèce de sous-titre à la photographie. Cette stratégie a été employée maintes fois dans la campagne parce qu'il était peu connu du grand public. Une typographie plus petite est aussi un signe plein, signifiant la minime importance du nom : peu importe qui est le candidat. Le plus important est le trait positif qui lui est associé : le courage - caractéristique valorisée par le parti pour ce poste.

Dans la partie verte apparaissent les deux symboles du parti et l'adresse du site. Quelles sont les diverses significations perçues en rapport avec ces éléments? L'un des symboles du parti (le poing serré) s'inscrit dans une tradition tandis que l'autre (la rose blanche, introduite dans la campagne électorale de 1992) dénote sa modernité. Quoique les deux symboles soient présents dans les affiches politiques utilisées en 1992 et 2002, nous observons une différence importante : tandis qu'en 1992, la rose blanche apparaît à gauche du poing serré, c'est ce dernier qui, en 2002, figure à sa gauche sur l'image. Cette reformulation de la posture du parti peut attester le besoin de récupérer les valeurs traditionnellement défendues par le socialisme portugais. Et le peuple portugais, en 2002, passe par des moments d'incertitude, de désespoir et a besoin de continuer à croire à l'idéologie socialiste. En revanche, la présence, sur l'affiche, de l'URL du site du parti montre aussi sa modernité : le parti s'adapte aux innovations technologiques.

Les couleurs du drapeau portugais, rouge et vert, qui apparaissent en dégradé à l'arrière-plan ont aussi une signification : témoigner du patriotisme du parti et de son engagement pour résoudre les problèmes du pays. De plus, le dégradé des couleurs suggère un drapeau en mouvement - ce qui peut signifier que le parti essaie de se transformer, de se moderniser. Le degré de modulation du vert que nous constatons dans cette affiche traduit le mouvement du parti et c'est exactement cette évolution idéologique qu'il veut transmettre.

Enfin, les procédés non verbaux (couleurs, typographie) peuvent même fonctionner, dans cet exemplaire de texte, comme des organisateurs textuels ${ }^{12}$. Par exemple, on peut penser que la couleur rouge, présente à peu près dans le tiers de l'affiche, isole l'argument de la thèse du texte. Par des mouvements inférentiels, nous pourrions établir des enchaînements argumentatifs : [Si vous

12. Cette expression a été proposée par Schneuwly, Rosat et Dolz (1989, p. 40-58). 
voulez quelqu'un de courageux (l'argument), il faut voter pour le PS - représenté par le poing serré, la rose et le site du parti (la thèse)]. Et les éléments qui traduisent la thèse du texte se situent dans la partie inférieure, verte, de l'affiche. De même la typographie de l'unité coragem est tout à fait différente des autres unités. Ce qui la distingue comme un argument au service de tout l'univers textuel.

Essayons maintenant de reconstituer quelques-unes des impressions transmises par la photographie du candidat ${ }^{13}$. Il importe de rappeler, comme nous l'avons déjà mentionné auparavant, que le mode sémiotique visuel, dans ce cas la photographie, peut être décrit à partir du signifié représentationnel, interactif et compositionnel. Comme notre intérêt porte surtout sur le type d'interaction établi entre les participants représentés (dans ce cas le candidat), les producteurs de l'image (les photographes de l'agence de marketing) et les spectateurs (le peuple qui va voter), nous allons aborder surtout le signifié interactif associé à ce portrait.

Comme nous le savons, d'après Kress et Van Leeuwen, trois éléments composent ce signifié : le contact, la distance sociale et l'attitude ou la perspective.

Dans l'affiche analysée, nous remarquons que le regard du candidat (participant représenté) est dirigé vers le spectateur (le public). À partir de ce regard direct, nous pouvons penser à l'existence d'une espèce de demande implicite du parti : [Votez pour ce candidat parce qu'il est honnête]. Par rapport à la distance sociale, nous observons que le candidat est cadré de façon relativement serrée : seul son buste apparaît, situé au premier plan. Ces aspects mettent en valeur, peut-être, la proximité entre cet homme et le peuple. Cet élément est aussi renforcé par la perspective choisie (plan frontal) et par un angle de prise de vue qui s'établit au même niveau que les spectateurs, ce qui transmet du candidat une vision réaliste et naturalise la scène. C'est l'image d'un candidat ouvert au dialogue et proche de chaque individu.

Tous ces aspects joints au costume sombre, aux lunettes, aux cheveux grisonnants et à l'austérité du visage ont dans notre civilisation occidentale une signification bien précise : ils dénotent le sérieux d'une personne. Nous pouvons même penser, à l'instar de Barthes (1964), à l'existence d'une espèce d'ancrage ${ }^{14}$ entre les aspects verbaux et non verbaux.

13. L'un des textes fondateurs sur le questionnement du mythe «naturel» photographique est l'œuvre de Barthes (1964), selon lequel ily a toujours, dans la photographie, des interventions de l'homme (cadrage, distance, lumière, etc.) qui appartiennent toutes au plan de la connotation. Pourtant, dans ce travail, situé plutôt dans une approche de sémiotique sociale, nous analysons la photographie selon les paramètres proposés par Kress et Van Leeuwen (2006).

14. Selon Barthes (1964), le rapport entre l'image et la parole (morceau de texte) peut être de deux types : soit d'ancrage - il y a alors une valeur substitutive entre les deux et l'image détient la charge informative (plus fréquent dans la photographie de presse et la publicité); soit de relais, la parole et l'image étant alors dans un rapport complémentaire (plus fréquent dans les bandes dessinées). 
Il ressort de ce qui a été décrit que le parti veut créer un ethos de crédibilité et en même temps un ethos «timidement » moderne (présence du site du parti). Cette affiche cherche à plaire aux électeurs qui sont attachés à la tradition du parti, mais aussi à ceux qui souhaitent sa modernisation. En outre, elle cherche à toucher les gens qui pensent que le courage, la transparence, l'aspect sérieux sont essentiels pour un candidat au poste de Premier ministre. Si l'on emprunte la terminologie employée par Aristote, c'est la confiance que cette construction du pathos veut transmettre et c'est par là que l'adhésion du spectateur est acquise. Bref, chaque détail de l'ethos choisi a un impact sur le pathos.

\section{Étude de cas 2}

La deuxième affiche choisie (image 2 en annexe) émane aussi du PS. Elle figure José Sócrates, candidat du parti, à gauche de la photo, pouce levé. Son image occupe presque la moitié du document. À droite, trois unités verbales apparaissent : «Dia 27 vote PS» («Le 27 votez PS»), «Avançar Portugal ( (Avancer/ Portugal»)15, "Juntos conseguimos» («Ensemble nous réussissons»), tandis que le poing serré (symbole du parti) apparaît en haut à droite après le premier énoncé. Analysons d'abord les aspects linguistiques puis les éléments non verbaux.

Comme l'affirme Charaudeau (1990, p. 55), «le niveau linguistique est l'un des lieux où les rapports interactifs entre l'énonciateur [...] et les destinataires sont nécessairement marqués». Dans ce document, nous constatons l'implication de l'énonciateur et du coénonciateur avec des marques caractéristiques de ce type de codage, comme l'utilisation des expressions indéfinies ou des formes personnelles à la première ou deuxième personne : "[juntos] consegui[mos]»; «Dia 27 vote PS». L'utilisation du présent de l'indicatif, avec une valeur inchoative, dans le premier énoncé, et du subjonctifà valeur injonctive dans le deuxième, contribue à conférer un caractère persuasif à l'affiche. Comme dans la plupart des affiches, cependant, la relation argumentative reste implicite. C'est le lecteur qui doit la construire. C'est un pathos complice qui est construit, à partir de l'ethos d'un locuteur déjà victorieux et fier de lui-même. Ce qui intéresse le plus dans cette affiche, c'est précisément le portrait du candidat. Il n'établit plus un contact direct avec l'électeur, puisqu'il ne demande plus directement son vote. Il est représenté, pouce levé, comme quelqu'un qui a déjà été élu par le peuple, qui a déjà obtenu la victoire. Le choix d’un angle oblique et de la contre-plongée corrobore cette représentation. C'est un personnage à dimension plutôt historique, un homme qui dépasse les intérêts pré-

15. L'absence de lien syntaxique entre les deux mots superposés est la même en portugais qu'en français; il en irait différemment si nous avions : «Para frente/Portugal : Portugal/en avant». 
sents et immédiats. C'est exactement cette image que le parti veut montrer au grand public. L'affiche combine en fait des informations divergentes : si, d'un côté, les unités verbales sollicitent le vote du peuple, de l'autre, le candidat souriant proclame déjà une possible victoire. Le poing serré, symbole du parti, apparaît tout seul ${ }^{16}$ (sans la rose) dans le coin supérieur droit de l'affiche. Ce sont les valeurs traditionnelles du parti qui sont présentes pour renforcer la lutte pour une société plus équilibrée.

La couleur, comme mode sémiotique, est aussi très importante dans cet univers textuel. Les couleurs du parti, le vert et le rouge, dominent le texte et symbolisent aussi celles du drapeau portugais. Toutefois, ce qu'il nous semble important de remarquer, c'est que les deux couleurs ne sont pas aussi tranchées qu'elles l'étaient en 2002 : un dégradé de rouge et de vert apparaît. Selon la sémiotique visuelle décrite par Kress et Van Leeuwen (2006), les couleurs portent des potentialités symboliques culturellement établies et peuvent être influencées par l'environnement multimodal dans lequel elles s'intègrent. $\mathrm{Si}$, dans nombre de pays, le rouge est fréquemment associé à la révolution, à la lutte, il a dans cette affiche un rôle tout à fait particulier : servir de fond à toutes les unités verbales détachées en blanc, ce qui contribue à donner une force persuasive à ces éléments. La thèse représentée par "Dia 27 vote PS» et les arguments qui la légitiment, "Juntos conseguimos» et "Avançar Portugal», sont dans la partie rouge de l'affiche. Le jeu des couleurs doit être mis en relation avec les autres unités pour que nous puissions vraiment interpréter sa valeur symbolique : la lutte doit toujours continuer, malgré la victoire presque certaine.

Dans les limites de ce travail, nous ne pourrons proposer que quelques réflexions qui devront être confirmées dans un corpus plus ample.

D'abord, la multimodalité présente dans le genre affiche électorale contribue à la complexité de ce genre textuel, puisque sa construction combine différents modes sémiotiques - des unités non verbales (photographie, typographie, dispositifs chromatiques) et des unités verbales. Tous ces modes sémiotiques ont des spécificités descriptives et interagissent pour la construction d'un tout signifiant. Cette dynamique multimodale constitue un défi méthodologique intéressant pour les chercheurs puisque, pour pouvoir la décrire, nous sommes obligés de convoquer des cadres théoriques différents.

De plus, nous avons pu constater, à partir d'une méthodologie descendante d'analyse, que le contexte sociopolitico-économique contraint le choix des aspects multimodaux utilisés dans les affiches pour atteindre le but visé par le parti.

16. Dans les autres affiches politiques du parti à l'époque, la rose était toujours présente à côté du poing serré, dans la partie inférieure des documents. 
La crise politique et sociale de 2002, dans le pays, a obligé le parti à récupérer plus nettement ses valeurs traditionnelles. En 2009, la campagne électorale du PS continue cette démarche, mais elle s'actualise, se modernise. Le slogan «Avançar Portugal » se fait présent dans toutes les affiches et est accompagné de l'image d'un candidat plus optimiste et souriant. En outre, le parti choisit des angles d'attaque bien actuels : les énergies renouvelables, l'amélioration de l'emploi et la hausse des investissements.

Mais, surtout, par les procédés interactifs choisis, nous avons remarqué que, dans la première affiche, c'était plutôt un ethos sérieux, honnête, qui était construit alors que, dans la deuxième, c'est surtout l'ethos d'un homme victorieux et fier de ses actes qui surgit. Et cette construction suscite chez le coénonciateur des affects et des sentiments distincts, comme le souligne Christian Plantin (2004, p. 173) : "La position qui est défendue ici est celle de l'indiscernabilité, selon laquelle il est impossible de construire un point de vue argumentatif sans y associer un affect. »

Ainsi, pour que nous puissions rendre compte de la véritable nature d'une affiche, il faut considérer qu'il s'agit d'un matériau interactif complexe qui relève d'un dialogisme inhérent à toute production communicative. Ce que nous constatons, c'est que les textes répondent à des attentes du public, anticipent des effets souhaités et peuvent devenir le point de départ d'autres échanges.

\section{Références}

BARthes Roland, 1964, "Rhétorique de l'image », Communications, n4 4, p. $25-42$.

BRONCKART Jean-Paul, 2008, «Genre de textes, types de discours et “degrés” de langue.

Hommage à François Rastier», Texto! Textes et cultures, vol.XIII, nº $1 / 2$, en ligne : http://www.revue-texto.net/index.php?id=86 (consulté le 31 mars 2012).

Charaudeau Patrick, 2008, «Des conditions d'analyse d'une campagne électorale. Conditions structurelles, conditions circonstancielles et stratégies discursives», La présidentielle 2007 au filtre des médias étrangers, C. Pineira-Tresmontant éd., Paris, L'Harmattan, p. 13-18.

HALLIDAY Michael A. K., 1985, An Introduction in Functional Grammar, Londres, Edward Arnold.

KLEIBER Georges, 1990, La sémantique du prototype. Catégories etsens lexical. Paris, PUF. KRESS Gunther, 1998, "Visual and verbal modes of representation in electronically mediated communication. The potencials of new forms of text», Page to Screen. Taking Literacy into the Electronic Era, I. Snyder éd., Londres, New York, Routledge, p. 53-79.

KRESS Gunther, VAN LeEUWEN Theo, 2002, «Colour as semiotic mode. Notes for a grammar of colour », Visual communication, n 1, p. 343-369.

- 2006, Reading Images. The Grammar of Visual Design, Londres-New York, Routledge. Lısı Marco, 2008, «Ao serviço do líder. As campanhas eleitorais do Partido Socialista », 
Análise social, vol.XLIII, $\mathrm{n}^{\circ}$ 3, en ligne : http://analisesocial.ics.ul.pt/documentos/ 1222271209Z8tQX9hr2Ep66LW4.pdf (consulté le 9 novembre 2012).

LuCIOLE Guillemin A. et al., 1991, La politique s'affiche. Les affiches de la politique, Paris, Didier Érudition.

PInTo Rosalice, 2010, Como argumentar e persuadir. Prática política, jurídica, jornalística, Lisbonne, Quid Juris.

Plantin Christian, 2004, "Ad passiones. Affects et logique dans l'argumentation», Práticas de investigação em análise do discurso. Actas do II encontro internacional de análise linguística do discurso, M. A. Marques, M. E. Pereira, R. Ramos éd., Braga, Universidade do Minho - Centro de estudos humanísticos, p.163-179.

SAINT-GEORGES Ingrid (DE), 2008, "La multimodalité et ses ressources pour l'enseignement-apprentissage ", Cahiers de la section des sciences de l'éducation, n ${ }^{117}$, «Vos mains sont intelligentes! Interactions en formation professionnelle initiale, L. Filliettaz, I. de Saint-Georges, B. Duc éd., Genève, Faculté de psychologie et des sciences de l'éducation, p.117-158.

SCHnEuwly Bernard, Rosat Marie-Claude, Dolz Joaquim, 1989, «Les organisateurs textuels dans quatre types de textes écrits (élèves de 9, 12, 14 ans) », Langue française, n०81, p. 40-58.

VAN LeEUWEN Theo, 2006, "Towards a semiotics of typography», Information Design Journal, no 14 (2), p.139-155.

- 2008, Introducing Social Semiotics, Londres, New York, Routledge. 


\section{Annexe}

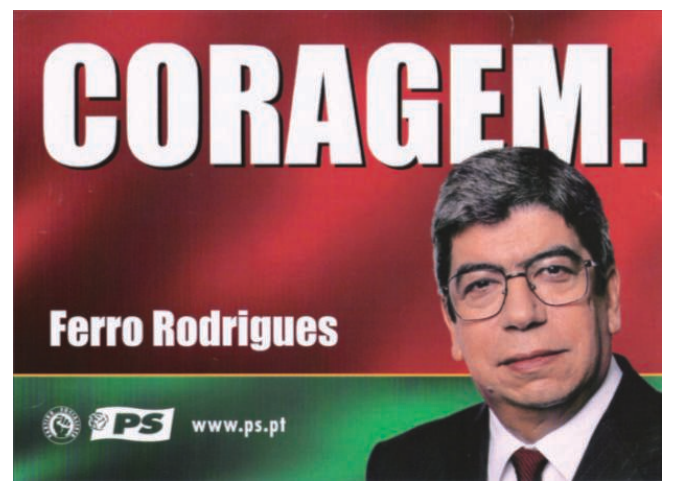

Étude de cas 1 : Affiche du Parti socialiste portugais, élections législatives de 2002

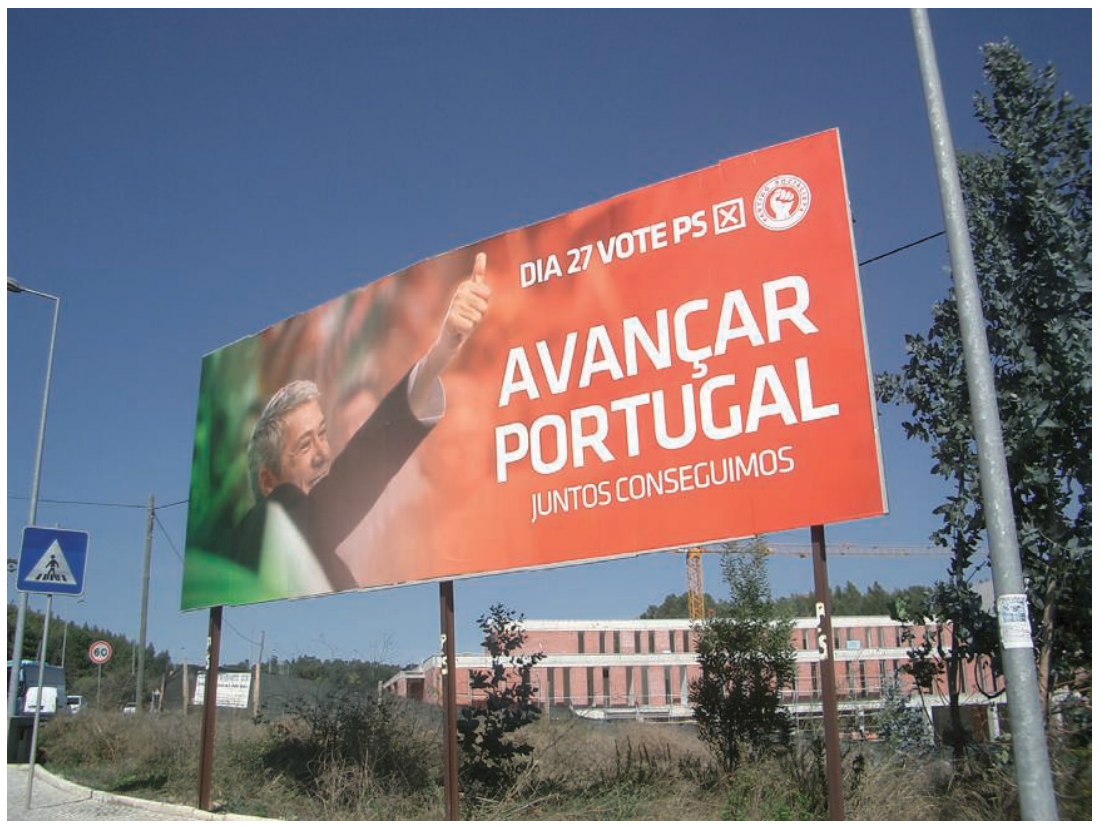

Étude de cas 2 : Affiche du Parti socialiste portugais (José Sócrates), élections législatives de 2009 